\title{
Template-based methodology for the simulation of intracorneal segment ring implantation in human corneas
}

\author{
Julio Flecha-Lescún · Begoña Calvo · Jesús Zurita · Miguel Ángel \\ Ariza-Gracia
}

Received: 22 November 2017 / Accepted: 12 March 2018

\begin{abstract}
Keratoconus is an idiopathic, non-inflammatory and degenerative corneal disease characterised by a loss of the organisation in the corneal collagen fibrils. As a result, keratoconic corneas present a localised thinning and conical protrusion with irregular astigmatism and high myopia that worsen visual acuity. Intracorneal ring segments (ICRS) are used in clinic to regularise the corneal surface and to prevent the disease from progressing. Unfortunately, the post-surgical effect of the ICRS is not explicitly accounted beforehand. Traditional treatments rely on population-based nomo-grams and the experience of the surgeon. In this vein, in silico models could be a clinical aid tool for clinicians to plan the intervention, or to test the postsurgical impact of different clinical scenarios. A semiau-
\end{abstract}

\section{Julio Flecha-Lescún}

Applied Mechanics and Bioengineering (AMB); Aragón Institute for Engineering Research (i3A), University of Zaragoza, Spain

Tel.: +34 876555492

E-mail: jflecha@unizar.es

\section{Begoña Calvo}

Applied Mechanics and Bioengineering (AMB); Aragón Institute for Engineering Research (i3A), University of Zaragoza, Spain.

Bioengineering, Biomaterials and Nanomedicine Online, Biomedical Research Center (CIBBER- BBN), Madrid, Spain

Jesús Zurita

Department of Mechanical Engineering, Public University of Navarra, Pamplona, Spain

Miguel Ángel Ariza-Gracia

Applied Mechanics and Bioengineering (AMB); Aragón Institute for Engineering Research (i3A), University of Zaragoza, Spain.

Computational Biomechanics (CB), Institute for Surgical Technology and Biomechanics (ISTB), University of Bern, Switzerland tomatic computational methodology is presented in order to simulate the ICRS surgical operation and to predict the post-surgical optical outcomes. For the sake of simplicity, circular cross section rings, average corneas, and an isotropic hyperelastic material are used. To determine whether the model behaves physiologically and to carry out a sensitivity analysis, a $3^{k}$ full-factorial analysis is carried out. In particular, how the stromal depth insertion (sDI), horizontal distance of ring insertion (hDRI) and diameter of the ring's cross-section $\left(\phi_{I C R S}\right)$ are impacting in the spherical and cylindrical power of the cornea is analysed. Afterwards, the kinematics, mechanics and optics of keratoconic corneas after the ICRS insertion is analysed. Based on the parametric study, we can conclude that our model follows clinical trends previously reported. In particular and although there is an improvement in defocus, all corneas presented a change in their optical aberrations. The stromal depth insertion is the parameter that affects the corneal optics the most, whereas hDRI and $\phi_{I C R S}$ are less important. Not only that, but it is almost impossible to achieve an optimal trade-off between spherical and cylindrical correction. Regarding the mechanical behaviour, inserting the rings at $65 \%$ depth or above will cause the cornea to slightly bend. This abnormal stress distribution greatly distorts the corneal optics and, more importantly, could be the cause of clinical problems such as corneal extrusion. Not only that, but our model also supports that rings are acting as restraint elements which relax the stresses of the corneal stroma in the cone of the disease. However, depending on the exact origin of the keratoconus, the insertion of rings could promote its evolution instead of preventing it. In conclusion, the methodology proposed is suitable for simulating long-term mechanical and optical effects of ICRS insertion. 
Keywords Corneal biomechanics - Template-based automatisation - Keratoconus - Intracorneal segment ring · Corneal ectasia · Finite element methodology

\section{Introduction}

Cornea is the outermost, transparent layer of the eye that is responsible of two-thirds of the optical power. It is structured in different layers that are the epithelium, the Bowmann layer, the stroma, and the endothelium, from the outermost to the innermost. Between them, the stroma represents almost the $90 \%$ of the corneal thickness and it is composed of water $(\approx 90 \%)$ and collagen fibres embedded in a ground substance called extracellular matrix (Garcia-Porta, 2014)(DelBuey, 2014, chap. 3). Collagen fibres, which are organised in a network, alongside with the extracellular matrix provide structural integrity and transparency to the cornea (Benoit, 2015).

Keratoconus (KC) is an idiopathic, non-inflammatory and degenerative corneal disease that typically develops in the inferior-temporal and central zones (Auffarth, 2000; Bao, 2016). Despite its ethiology is still partially unknown (Alio, 2017, chap. 3)(Alio, 2017, chap. 4) (Alio, 2017, chap. 12)(Alio, 2017, chap. 19), corneas with keratoconus or ectasia present a loss of organisation in the corneal collagen fibrils that results in a localised thinning and a conical protrusion (Auffarth, 2000). In KC, the corneal surface presents a progressive asymmetric deformation with irregular astigmatism and high myopia that worsens visual acuity (Bao, 2016). KC is ethnic-dependent (Alio, 2017, chap. 3) and targets on young to mid-age patients, appearing during the adolescence and progressing until maturity (Alio, 2017, chap. 19). Although its incidence is low, 0.05$2.5 \%$, the absence of a cure and its long-term blinding effects put $\mathrm{KC}$ on the spot.

In clinic, $\mathrm{KC}$ is managed using non-invasive and invasive treatments depending on the severity of the ectasia. In advanced ectasias that present severe astigmatism, elevated myopia or opacity of the stroma, surgeons are forced to use surgical techniques such as the instrastromal corneal ring segments (ICRS) (Akaishi, 2004; Zare, 2007) to avoid carrying out a penetrating keratoplasty or corneal graft (Gomes, 2015; VegaEstrada, 2016). ICRS have different objectives such as reinforcing diseased corneas without weakening the structural integrity(Garcia-Porta, 2014), regularising the shape of the corneal surface, and halting the progression of the cone which is suggested to be stress-driven (Bao, 2016; Vega-Estrada, 2016)(Alio, 2017, chap. 14). Several clinical studies (Vega-Estrada, 2016; Alio, 2005, 2006; JosephColin, 2001; Piñero, 2009; Shabayek, 2007) have demonstrated the effectiveness of ICRS treatment in correcting keratoconus and mild to moderate myopia (Suiter, 2000) using both INTACS micro-thing prescription inserts (Addition Technology, Inc., Sunnyvale, CA, USA) and Ferrara Rings (Ferrara Ophthalmics, Brazil) (Waleed, 2016; Fernandez-Vega, 2016; Jadidi, 2014; Torquetti, 2016).

Their main clinical setback is related to the planning of the clinical procedure. Treatments usually rely on the experience of the ophthalmologist (Alio, 2006; Fernandez-Vega, 2016) and population-based nomograms that implicitly account for the mechanics, but explicit patient-specific information is missing. Hence, unexpected outcomes can arise such as corneal extrusion in which, from 24 hours to few weeks after surgery (Liu, 2015; Zare, 2007), ICRS are expelled out the cornea, or imprecise post-surgical visual acuity that results in patient discomfort (JosephColin, 2012; Torquetti, 2013).

Rings are surgically placed depending on the location and degree of severity of the ectasia. Different surgical parameters must be accounted for to achieve the desired effect such as the distance from the corneal center where rings will be placed, the diameter and shape of the ring's cross-section, the angle embraced, or whether single or paired ICRS should be inserted (Vega-Estrada, 2016; Kahn, 2016; Kling, 2013). In actual practice, both triangular and hexagonal cross-sectioned ICRS are used (Piñero, 2009; Shabayek, 2007). The former are inserted close to the optical axis whereas the latter are inserted at a larger distances due to their bigger cross-section.

The surgical intervention consists of five steps (Coskunseven, 2008). First, a dissection plane, which is usually arch-shaped, is generated by a femtosecond laser in the peripheral region of the cornea at $70 \%$ or $80 \%$ of the stromal depth. Second, the dissection plane is visually cleared. Third, ICRS are manually implanted under full aseptic condition with a special guidance tools. Fourth, the final position of the segments is fine-tuned with a Sinskey hook using the dialling holes at both ends of the ICRS. Surgeons could take advantage of computational simulations in planning the surgery, or in providing qualitative post-surgical information in different surgical scenarios such as the residual astigmatism that will arise from the intervention, or if the ICRS will stabilise the progression of the keratoconus.

Few computational studies have been addressed to simulate the surgery (Kahn, 2016; Kling, 2013; Lago, 2015)(Guarnieri, 2015, chap. 14) In particular, two works convey the main modelling approaches adopted for tackling the ICRS simulation. First, Kling et al. (2013) analysed the effect of the insertion of ICRS in average healthy and ectatic corneas. A 2D axisymmetric model was used including the external structures of the eye- 
ball, i.e., cornea, limbus and sclera, and triangular and hexagonal ICRS. The material behaviour was assumed as hyperelastic and isotropic for all the ocular tissues. For the insertion of the rings, a previous gap was included in the pre-surgical configuration of the cornea. Besides, the pre-stress of the ocular tissues due to the intraocular pressure was not accounted for.

Second, Lago et al. (2015) build a patient-specific model of the cornea without additional structures (i.e., limbus or sclera) to predict the post-surgical effects of ICRS. As boundary condition, the displacements of the corneal periphery were restrained. The material behaviour was also assumed as hyperelastic and isotropic and included the pre-stress of the ocular tissues. For the insertion of the rings, a previous gap was included in the pre-surgical configuration of the cornea and the ICRS were not simulated as a solid inside the cornea but the nodes of the gap were morphed into the shape of the ring instead.

Despite the interest and novelty of these methodologies, two main points could be improved: i) a mechanical weakness is introduced beforehand when considering the gap of the tunnel from the beginning, which could be inaccurate when representing the mechanical equilibrium prior to the surgery; ii) the solid rigid motion of the ICRS after its insertion is missing if the rings are not represented as a foreign body inserted in the incision and interacting with the surrounding tissue.

In the present work, we present the first semi-automatic tool that is template-based to simulate the 3D implantation of ICRS in human corneas. Our numerical framework allows for simulating the optical and mechanical effect of three different key parameters of the ICRS surgery that are the stromal depth at which the ring is inserted (sDI - stromal depth of insertion), the horizontal distance with respect to the corneal center where the rings are placed (hDRI - horizontal distance of ring insertion), and the diameter of the cross-section $\left(\phi_{I C R S}\right)$. Besides, the pretension of the tissues is included (Ariza-Gracia, 2016), the gap associated to the laser incision is not present in the pre-surgical configuration of the cornea, and the rings are introduced as solid bodies that contact with the corneal stroma.

For the sake of simplicity, and since the target of this work is to show a numerical methodology, we assume three main simplifications: the use of an average human cornea (Navarro, 2006), the description of the cornea as a hyperelastic isotropic material (Ariza-Gracia, 2016), and the use of ICRS with circular cross-section, which are seldom applied in clinic. Independently of these simplifications, the procedure remains valid whether more complex scenarios are introduced, e.g., patient-specific corneas, anisotropy of the tissues, or other ICRS crosssections such as triangles or hexagons.

To determine whether our computational model is behaving physiologically, we perform a $3^{k}$ full-factorial parametric study (Montgomery, 2001) that analyses the impact of these three key parameters (i.e, sDI, hDRI, and $\left.\phi_{I C R S}\right)$ in the optics, the mechanics and the kinematics of the cornea. Using an in-house ray-tracing algorithm (Ariza-Gracia, 2017), we also analyse the optical effect in the post-surgical cornea including the aberrations of the eye related to the Zernike coefficients, and the spherical and cylindrical powers, which are typically used to assess in the visual acuity of the patient. Based on this $3^{k}$ parametric study, we also try to discern the most influential parameters that affect patient's optics.

Finally, the methodology is applied to an average cornea that presents a paracentral keratoconus to determine whether the methodology can be further applied to assess in the management of the disease. The kinematics, the mechanics and the optics of the postsurgical cornea are analysed for two different ring diameters $\left(\phi_{I C R S}\right)$ and two different stromal depths of insertion (sDI).

\section{Material and Methods}

In this section, the template-based computational methodology is presented. In particular, the methodology gathers the following aspects: i) the definition of the average finite element templates and their numerical features, i.e., mesh, boundary conditions and material behaviour; ii) the definition of the finite element model of the ICRS; iii) the computational procedure proposed to simulate the long-term insertion of the ICRS in the cornea; and $i v$ ) the optical criteria used for analysing the results.

Then, a $3^{k}$ full-factorial parametric analysis is used to study the influence of different ICRS's parameters in the corneal optics, and whether our models and procedure yield physiological outcomes. Finally, a theoretical study in keratoconus (KC) corneas is performed to discern whether the computational framework could help in its management by determining the mechanical and kinematic response of the cornea after the insertion of ICRS.

$2.13 \mathrm{D}$ finite element template of the human eyeball: mesh, constitutive behaviour, and boundary conditions

Two geometries of reference are considered to build the finite element (FE) templates: an average healthy 
cornea and an average cornea with paracentral keratoconus. All structures in the model, i.e., cornea, sclera and limbus, present symmetry of revolution (see in figure 1.a). The dimensions of the healthy cornea are based in an emmetropic eye (Navarro, 2006): radio of curvature of $6.7 \mathrm{~mm}$ and $7.5 \mathrm{~mm}$ for the posterior and anterior surfaces respectively, and a variable and incremental thickness from the center to the periphery with a fixed central corneal thickness of 600 microns. The paracentral keratoconus is derived from the healthy cornea simulating that the healthy patient develops the disease. It has a central thinning of 400 microns, and a mechanical weakening that is affecting $2 \mathrm{~mm}$ in diameter (see in figure 1.b). The inner diameter of the sclera is set to $24 \mathrm{~mm}$ (Navarro, 2006) with a constant thickness of $1 \mathrm{~mm}$. The limbus is defined as the short strip of tissue that acts as transition between the cornea and the sclera.

Nine templates are build by combining three variations of hDRI, i.e., 5,6 , and $7 \mathrm{~mm}$, and three variations of sDI, i.e., $50 \%, 65 \%$, and $85 \%$ (see in figure 1.b). All geometries are meshed with 8-node linear hybrid hexahedral elements $(\mathrm{C} 3 \mathrm{D} 8 \mathrm{H})$. To determine the optimal size of the elements, a convergence analysis of the mesh has been carried out (results not shown). As the problem is dominated by the biaxial stress state induced by the intraocular pressure (IOP) and there is no bending (Ariza-Gracia, 2016), the mesh size at the center of the cornea is not critical. However, areas close to the insertion of the ICRS undergo great deformation and, thus, precise of a fine mesh definition. Hence, the cornea is overmeshed in some regions to avoid a bad mesh transition between the fine mesh surrounding the ICRS and the ideal coarse mesh in the rest of the cornea. Depending on the combination under analysis, the number of elements of the mesh will vary from 184,572 to 311,052 elements, i.e., from 773,055 to 977,604 degrees of freedom (D.O.F).

To simulate the material behaviour of the ocular tissues, we use hyperelastic isotropic strain-energy functions. Despite the fact that the human cornea presents two orthogonal families of fibres (Ariza-Gracia, 2016; Pandolfi, 2011), the limbus presents one circumferential family of fibres (Ariza-Gracia, 2016; Pandolfi, 2011), and the sclera presents a random distribution of fibres far from the optical nerve insertion (Coudrillier, 2015), they are not modelled as fibre-reinforced materials. This assumption does not affect the proposed methodology to simulate the insertion of the ICRS, but allows for a less time-consuming analysis. While the cornea and the limbus are modelled using Neo-Hookean strain-energy functions, $\psi_{N}$, (Ariza-Gracia, 2015) the sclera is mod- elled using a Yeoh strain-energy function, $\psi_{Y}$ (ArizaGracia, 2016).

$$
\begin{aligned}
& \psi_{N}=\frac{1}{D} \cdot\left(\frac{J_{e l}^{2}-1}{2}-\ln \left(J_{e l}\right)\right)+C_{10}^{N} \cdot\left(\bar{I}_{1}-3\right) \\
& \psi_{Y}=\sum_{i=1}^{3} D_{i} \cdot\left(J_{e l}-1\right)^{2 \cdot i}+\sum_{i=1}^{3} C_{i 0}^{Y} \cdot\left(\bar{I}_{1}-3\right)^{i}
\end{aligned}
$$

where $\bar{I}_{1}$ is the first invariant of the modified right Cauchy-Green tensor $\bar{C}=J_{e l}^{-2 / 3} C, J_{e l}$ is the elastic volumen ratio, and $\frac{1}{D_{i}}$ is the bulk modulus. The material constants for the healthy tissues are retrieved from our previous work (Ariza-Gracia, 2016): $C_{10}^{N}=0.05 \mathrm{MPa}$, $C_{10}^{Y}=0.81 \mathrm{MPa}, C_{20}^{Y}=56.050 \mathrm{MPa}, C_{30}^{Y}=2,332.26 \mathrm{MPa}$, $D_{i}=0 \mathrm{MPa}^{-1}$. The material weakening associated to the $\mathrm{KC}$ is assumed to be a $50 \%$ of the stiffness of the healthy material: $C_{10}^{K C}=0.025 \mathrm{MPa}$.

As model-dependent boundary conditions, i.e., not related to the surgical procedure, there are two main restrictions: the intraocular pressure (IOP) that is set to a physiological pressure of $15 \mathrm{mmHg}(2 \mathrm{kPa})$, and a condition of symmetry on the equatorial plane of the sclera (Ariza-Gracia, 2016). Moreover, the initial prestress of the corneal tissue is introduced by using an iterative algorithm previously reported (Ariza-Gracia, 2016). The free-stress algorithm determines a reference geometry, generally smaller, such that when the eyeball is pressurised to the physiological IOP, the corneal shape returns to its original shape but including the stress and stretch fields.

\section{$2.23 \mathrm{D}$ finite element model of the ICRS}

For the sake of simplicity and numerical stability, ICRS with three different circular cross-section diameters $\left(\phi_{I C R S}\right.$ $=200,300$ and 500 microns) and a fixed opening angle, $\alpha$, of $150^{\circ}$ are used. The diameter of the circular rings corresponds to the maximum limiting dimension of clinical rings (i.e., triangular or hexagonal, see in figure 2). In this vein, we ensure that the minimum size of the laser incision will house any ICRS typology, even when commercial triangular or hexagonal ICRS geometries are introduced.

ICRS are made of PMMA isotropic polymer and modelled as an elastic material with a Young modulus of 3,300 $\mathrm{MPa}$ and a Poisson's ratio of 0.4 (data from Addition Technology, Inc., Sunnyvale, CA, USA). They are meshed using 8-node linear hexahedra (C3D8) with a variable number of elements ranging from 3,343 to 9,024, i.e., from 12,783 to 32,460 DOF. Regarding their model-dependent boundary conditions, there are not special considerations as they will lay over the cornea 


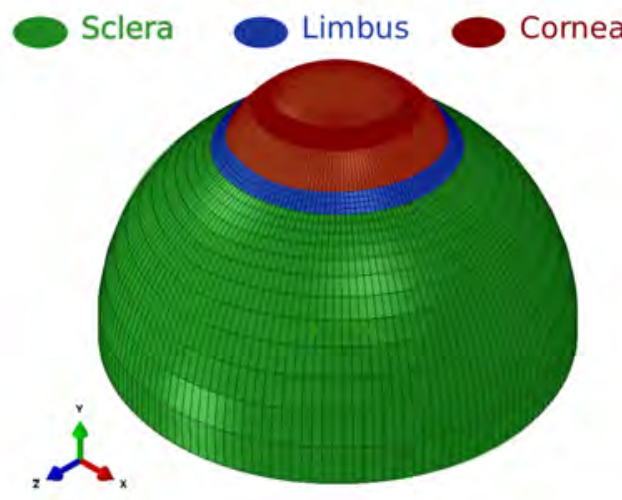

(a)

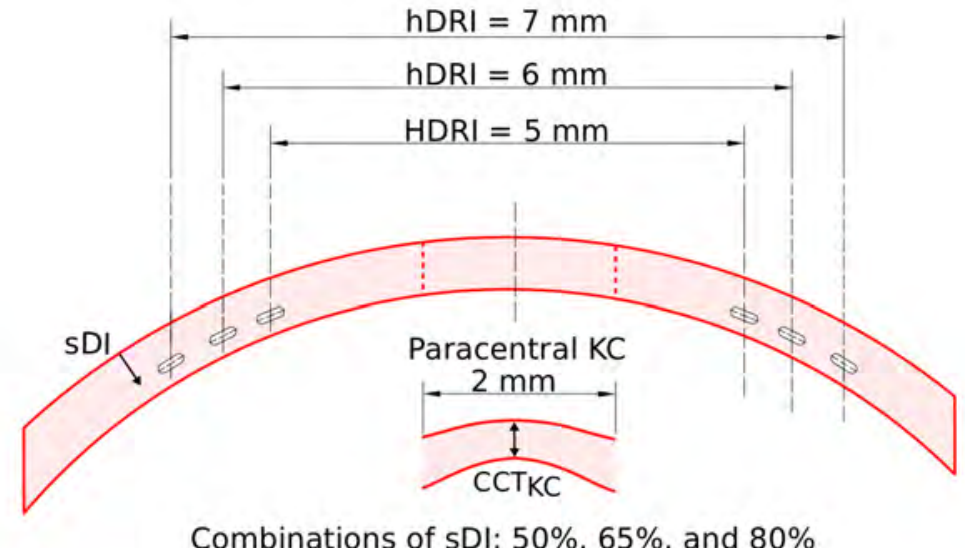

(b)

Fig. 1 Definition of the finite element templates: (a) 3D numerical model of the eyeball including cornea (red), limbus (blue), and sclera (green); (b) Schematic transversal section of the cornea highlighting two of the variables controlled in the template: stromal depth of insertion (sDI), and horizontal distance of ring insertion (hDRI). Detail of KC geometry with a central thickness $\left(\mathrm{CCT}_{\mathrm{KC}}\right)$ of 400 microns

until their insertion inside the laser incision. Once inside, a frictionless, hard-contact, surface-to-surface contact between the rings and the incision is activated.

\subsection{Computational simulation of the insertion of ICRS}

Three stages of the surgery must be simulated in Abaqus (Dassault Systèmes Simulia Corporation) to mimic the surgical procedure: $i$ ) the creation of the laser incision in the stroma, ii) the widening of the incision to introduce the rings, and iii) the insertion of the ICRS. Since our interest is in the long-term impact of the ICRS in the mechanics and the optics of the cornea, and not in the damage mechanisms of the photodisruption, incisions are already "included" in the FE template but "filled" with corneal stroma (see figures 1 and 3). In this way, the pre-stress of the pre-surgical geometry of the cornea can be done without introducing a fictitious weakening due to the gap (Kahn, 2016; Kling, 2013; Lago, 2015). After pre-stressing the cornea, the elements of the incision are removed using the Abaqus Standard tool, *Model Change, that allows erasing elements during a simulation step. As a mechanical instability is introduced, a new mechanical equilibrium needs to be achieved.

Once the incision is empty, it is enlarged using a pressurisation to give room for the ICRS. In particular, the *Fluid Cavity boundary condition that allows for introducing a hydrostatic pressure is used. This hydrostatic pressure can be either controlled by fixating the pressure degree of freedom, or by introducing a vol- ume of fluid in the cavity. Since, the amount of fluid to introduce is not known beforehand, the pressure is fixed and the volume flowing in the cavity is monitored (see in figure 3). When the incisions are expanded, their center lines are calculated and the rings are registered into the correct position. Finally, the pressure is slowly released allowing the incision to shrink and to establish the contact with the ICRS.

There are two critical computational restrictions that must be satisfied to obtain the convergence of the problem. First, the incision must be sensitively bigger than the diameter of the ICRS $\left(\phi_{I C R S}\right)$ or the surface normals will not cross, the contact will not be detected, and meshes will penetrate. Second, different levels of pressure are needed to obtain the same volume inside the cavity depending on the material stiffness of the area surrounding the incision, the hDRI, and the sDI. A preliminary analysis is carried out to define the level of pressure needed to open the incision and to insert the ICRS without violating any of the computational restrictions. To allow the insertion of the different ICRS at different sDIs and hDRIs, the following levels of pressure are needed: i) at $50 \%$ depth, $400 \mathrm{kPa}$ with an average volume of $5.035 \pm 0.569 \mathrm{~mm}^{3}$; ii) at $65 \%$ depth, $385 \mathrm{kPa}$ with an average volume of $5.034 \pm 0.605 \mathrm{~mm}^{3}$; iii) at $80 \%$ depth, $275 \mathrm{kPa}$ with an average volume of $4.774 \pm 0.494 \mathrm{~mm}^{3}$.

The complete procedure (see in figure 3 ) consists of the following steps: $i$ ) determining the free-stress configuration of the cornea (1st simulation); ii) physiological pre-stressing of the cornea due to the intraocular pressure (2nd simulation); iii) removing the elements 


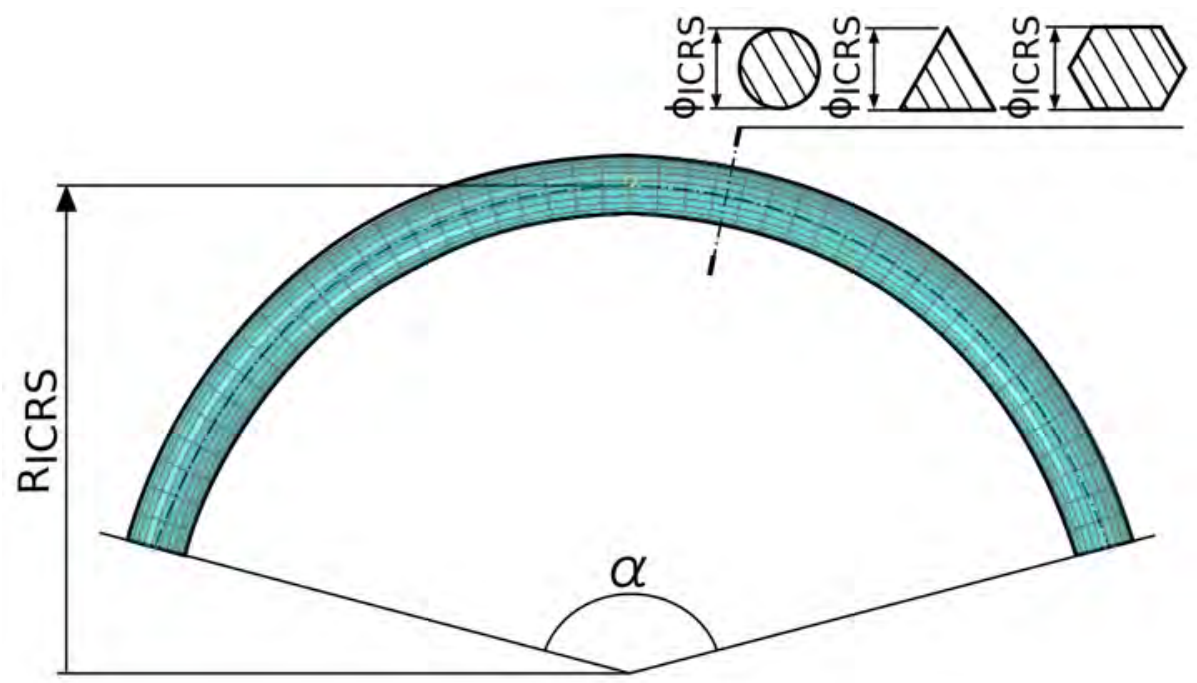

Fig. 2 Finite element model of the ICRS. The main variables are the radius of the ring $\left(R_{I C R S}\right)$, the opening angle $(\alpha)$, and the diameter of the transverse section of the ring $\left(\phi_{I C R S}\right.$. The diameter of the circular ring is set to the maximum limiting dimension of commercial rings (triangular and hexagonal)

inside the laser incision (2nd simulation); iv) expanding the incisions (2nd simulation); v) calculating the center lines of the incisions (2nd simulation); vi) registering the ICRS to the incisions (3rd simulation); vii) closing up the incisions to force contact with the ICRS (3rd simulation); viii) achieving the mechanical stability (equilibrium step).

During the simulation, the elevation of the anterior and posterior corneal surfaces are saved. For the preand post-surgical geometries, the Cartesian coordinates $(\mathrm{x}, \mathrm{y}, \mathrm{z})$ of the points defining the anterior and posterior surfaces are saved to a text file using the URDFIL subroutine. With this information, the optical assessment of the pre- and post-surgical influence of the ICRS is carried out.

\subsection{Determination of corneal optics}

To use metrics that are of clinical relevance, our inhouse ray-tracing software (Ariza-Gracia, 2017) is used. Such software allows for the numerical simulation of clinical topographers (Bao, 2016; Garzon, 2013; Hong, 2013) and aberrometers (Piñero, 2009; Lakshminarayanan, 2011) using as input information the corneal elevation of both surfaces.

In particular, we use the concept of wavefront aberration in optical systems (Dai, 2008; Malacara, 2003). The wavefront aberration is the measure of the degree of imperfection of the optical system: while in a perfect system planar waves transform into perfect spherical waves, in real systems the same planar waves would transform into distorted spheres. This distortion gives direct information of the imperfections of the system, either in geometry or in the transmission of the light, i.e., refraction indexes. Importantly, the wavefront aberration can be represented as a surface and, thus, it can be fitted to Zernike polynomials (Lakshminaraya-nan, 2011).

These polynomials have a direct relation to clinical practice since some of their coefficients $\left(Z_{n}^{m}\right)$ are indicator of common pathologies such as astigmatism, which is related to $Z_{2}^{-2}$ and $Z_{2}^{2}$, or keratoconus, which is related to $Z_{1}^{-3}$ and $Z_{1}^{3}$ (see brief summary in figure 4).

In ophthalmology, the spherical (Sph) and cylindrical (Cyl) powers (diopters, D) are used to assess on the visual acuity of the patient, for example in the prescription of lenses (Thibos, 2004). Patient's astigmatism can be determined with the second-order coefficients of Zernike, i.e., $Z_{2}^{-2}, Z_{2}^{0}$, and $Z_{2}^{2}$, by calculating the cylindrical power and the axis of orientation of the cylinder $(\phi)$ (Eq.3) (Thibos, 2004):

$$
\begin{array}{r}
S p h(D)=\frac{-Z_{2}^{0} 4 \sqrt{3}}{R_{p}^{2}}-\frac{C y l}{2} \\
\operatorname{Cyl}(D)=-2 \cdot \sqrt{\left(\frac{-Z_{2}^{2} 2 \sqrt{6}}{R_{p}^{2}}\right)^{2}+\left(\frac{-Z_{2}^{-2} 2 \sqrt{6}}{R_{p}^{2}}\right)^{2}} \\
\phi\left(^{o}\right)=\frac{1}{2} \operatorname{atan}\left(\frac{\frac{-Z_{2}^{-2} 2 \sqrt{6}}{R_{p}^{2}}}{\frac{-Z_{2}^{2} 2 \sqrt{6}}{R_{p}^{2}}}\right)
\end{array}
$$

where $R_{p}$ is the radius of the pupil under analysis, i.e., typically $3 \mathrm{~mm}$ (Hernandez-Gomez, 2014). 

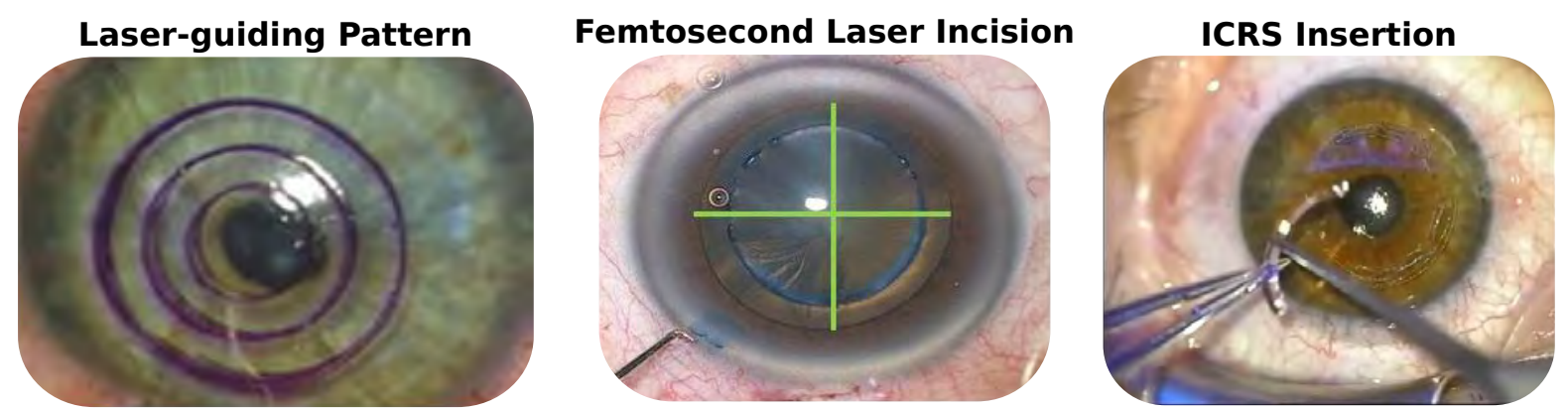

(a)
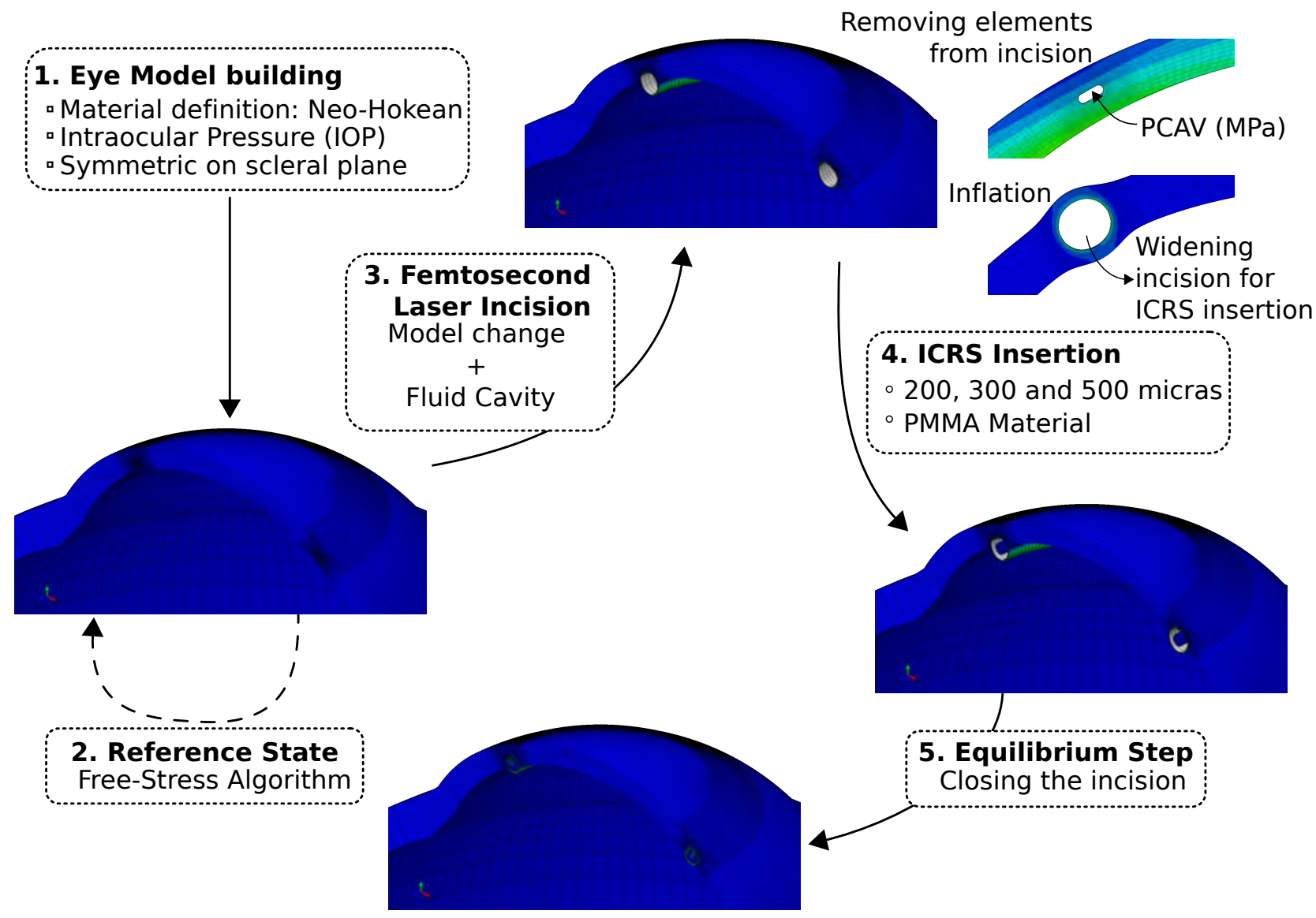

(b)

Fig. 3 Clinical and computational surgical procedure: (a) Clinical procedure: a pattern is marked so as to the laser can perform the incision and the rings can be manually inserted; (b) Computational procedure: (1) the numerical template is build; (2) The reference configuration is achieved and the ocular tissues are pre-stressed using an iterative algorithm (Ariza-Gracia, 2016); (3) the incisions, which were already present in the model, are removed using ${ }^{*}$ Model Change and inflated using ${ }^{*}$ Fluid Cavity; (4) the ICRS is inserted and the pressure slowly released; (5) the ICRS reaches the equilibrium inside the incision. A video of this surgery simulation is attached. The numerical methodology is also reported in the video provided as supplementary material (upper left panel). In particular, the KC cornea with a 200 -micron ICRS inserted at $80 \%$ depth is presented 


\begin{tabular}{ccc|c}
$i$ & $n$ & $m$ & Name \\
\hline 1 & 0 & 0 & Piston \\
2 & 1 & -1 & Tilt \\
3 & 1 & 1 & Tip \\
4 & 2 & -2 & Oblique astigmatism \\
5 & 2 & 0 & Defocus \\
6 & 2 & 2 & Vertical astigmatism \\
7 & 3 & -1 & Vertical Coma \\
8 & 3 & 1 & Horizontal Coma
\end{tabular}

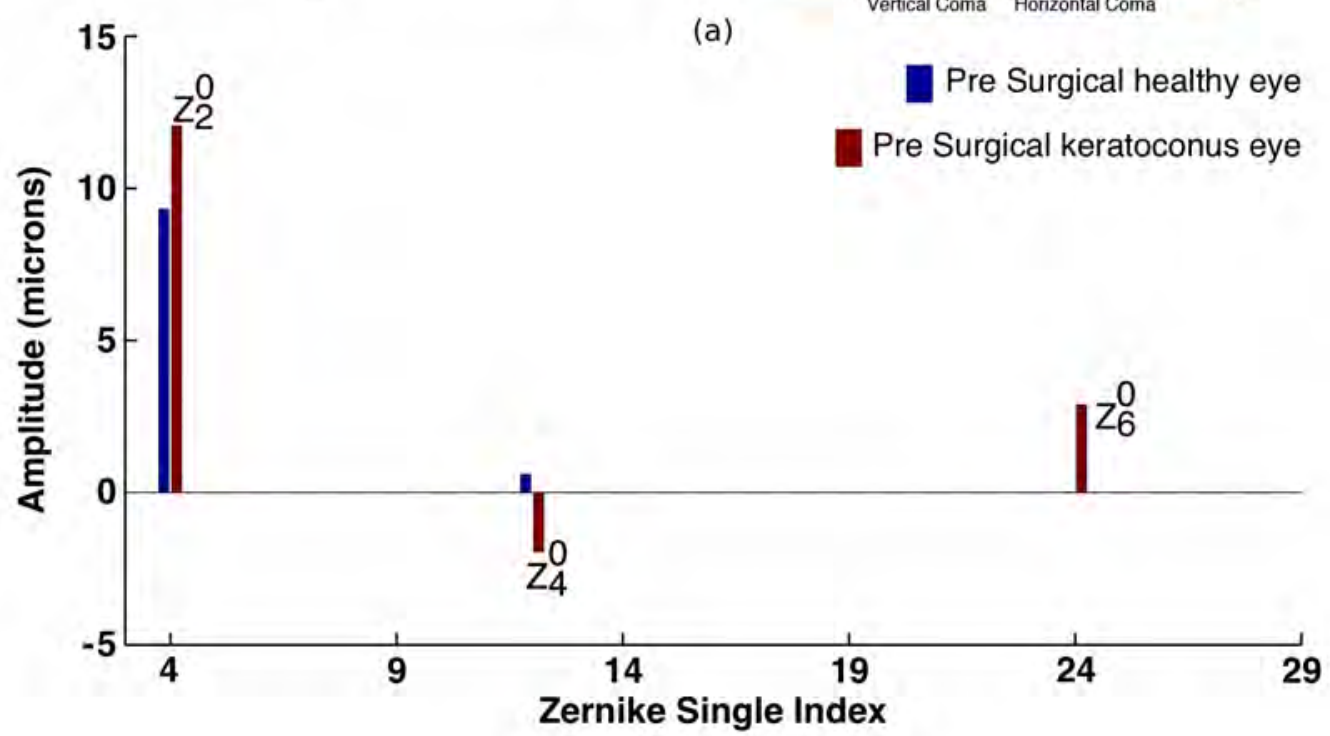

(b)

Fig. 4 Zernike representation of the geometries of reference: (a) summary of the most representative Zernike coefficients $\left(Z_{m}^{n}\right)$ and their graphical representation. The cylindrical power (astigmatism) and the spherical power (defocus) are related to the second-order Zernike coefficients $\left(Z_{2}^{-2}\right.$-oblique astigmatism, $Z_{2}^{0}$-defocus, and $Z_{2}^{2}$-vertical astigmatism). Keratoconus is related to vertical and horizontal coma $\left(Z_{1}^{-3}\right.$ and $\left.Z_{1}^{3}\right)$; (b) Zernike coefficients of the average healthy (blue) and average KC (red) geometries. The healthy geometry only presents primary and secondary spherical aberrations $\left(Z_{2}^{0}\right.$ and $\left.Z_{4}^{0}\right)$. The KC geometry, as it is derived from the healthy geometry, worsens the primary and secondary spherical aberrations and introduces high order spherical aberrations $\left(Z_{6}^{0}\right)$. Moreover, astigmatism is not present as the $\mathrm{KC}$ is of paracentral type

The optical outcomes of the FE templates are gathered in figure 4.b. The initial average cornea presents primary and secondary spherical aberrations $\left(Z_{2}^{0}\right.$, and $Z_{4}^{0}$ ), whereas the evolution of the paracentral $\mathrm{KC}$ worsens the previous aberrations $\left(Z_{2}^{0}\right.$, and $\left.Z_{4}^{0}\right)$ and promotes new high order aberrations $\left(Z_{6}^{0}\right)$. As the case of study is a theoretical perfect paracentral $\mathrm{KC}$, primary astigmatism $\left(Z_{2}^{-2}\right.$, and $\left.Z_{2}^{2}\right)$ is not present in the relation of aberrations.

\subsection{Benchmark studies}

A $3^{k}$ full-factorial protocol is used to design a batch of experiments that is based on the FE templates of the healthy cornea and in the in silico surgical methodology. This parametric study presents 3 levels (low, mid, high) of combinations of $k$ variables to assess in the impact of one, or several, objective variables (Montgomery, 2001). In particular, our parametric study is build using a $3^{3}$ full-factorial analysis with 27 simulations that combines three levels of: sDI (50\%, $65 \%$ and $80 \%)$, hDRI ( 5,6 and $7 \mathrm{~mm}$ ), and $\phi_{I C R S}(200,300$, and 500 microns). 
With this parametric study, we analyse the impact of different clinical scenarios on two optical variables: the spherical power (Sph), and the cylindrical power $(\mathrm{Cyl})$. Note that the astigmatic axis $(\phi)$ was not analysed since we are using average corneas and rings are always placed in the same corneal meridian. Thus, the astigmatic axis will always align with the ICRS axis and will not present variation between clinical scenarios. Thanks to the level-wise structure of the dataset, the main effect of the principal variables and their interaction on the optical response is analysed (Montgomery, 2001).

Finally, the kinematics, the mechanics, and the optics are studied for both the healthy and $\mathrm{KC}$ corneas. In particular, three cases are analysed: i) Case 1: 200 micron ICRS placed at 80\% depth; ii) Case 2: 500 micron ICRS placed at $80 \%$ depth; and iii) Case 3: 200 micron ICRS placed at $50 \%$ depth. The main outcomes of interest are: the movement of the corneal apex, the stress field in the corneal stroma next to the ring, the stress relaxation of the anterior and the posterior cone, and the corneal optics.

FE simulations were performed using Abaqus, whereas the optical analysis and data management were performed using Matlab (Matrix Laboratory, Mathworks).

\section{Results and Discussion}

In this section, benchmark studies are discussed. First, the parametric study used to discern whether the model behaves physiologically and to study the impact of different ICRS features on corneal optics. Second, the kinematic, mechanical, and optical analysis of different clinical scenarios in KC corneas. For the sake of clarity, only the results for $\mathrm{KC}$ corneas are showed since both corneal configurations, healthy and $\mathrm{KC}$, behaved similarly.

\subsection{Results of the parametric study}

The most influential parameter of the surgery is the stromal depth of insertion (sDI). The impact of the variation of the sDI represents slightly more than a $70 \%$ of the variation of spherical power and more than a $50 \%$ of the variation in cylindrical power (see in figure 5.a-b). This is in line with several clinical studies where depth was reported to be critical in the definition of the ICRS surgery (Waleed, 2016; Fernandez-Vega, 2016; Jadidi, 2014; Torquetti, 2016). The deeper the location of the ICRS, the greater the impact in both powers but, interestingly, with opposite slopes (see in figure 5.c-d). This is essential when performing a surgery since there is not an optimal trade-off when minimising aberrations: if the spherical aberration aims at being minimised, the astigmatism (cylindrical power) will increase and viceversa. Physically, this means that the curvature of the surface is greatly affected due to the kinematics and mechanics of the cornea after the insertion of the ring. As we consider the material fixed, we do not know exactly how much of this contribution is derived from the material or the shape, but we can infer that mechanics is playing a role that is driving the response. Furthermore, the cylindrical power presents a nonlinear behaviour with a transition zone at $65 \%$ depth from which there will be negligible changes (see sDI in figure 5.d).

The influence of the hDRI ranges from 10 to $20 \%$ for the spherical and cylindrical powers, respectively (see in figure 5). Also this feature affects the spherical and cylindrical powers with opposite linear trends, being impossible to obtain an optimal trade-off between both powers when performing a surgery. For example, if the astigmatism wanted to be minimised, the optimal hDRI would lie between 6 and $7 \mathrm{~mm}$, but this would imply that a certain amount of spherical aberration would be induced.

The diameter of the ICRS $\left(\phi_{I C R S}\right)$ only affects the spherical power whereas the cylindrical power presents much less sensitivity to it (see in figure 5.a-b). Besides, both power could be minimised in terms of $\phi_{I C R S}$ as they present linear positive trends (see in figure 5.c-d). At this point, we must stress that the ICRS with a diameter of 500 micros was calculated but removed from the statistical analysis as they resulted in anomalous values and distorted the analysis. In fact, the inclusion of such rings is related to the $3^{k}$ full-factorial analysis where the levels of variation must be fixed and complete. Nevertheless, this is not affecting either the conclusions or the results, since such ICRS are scarcely used in clinics and always far away the center (i.e., $>7 \mathrm{~mm}$ )(Waleed, 2016; Fernandez-Vega, 2016; Jadidi, 2014; Torquetti, 2016).

When observing the interaction between ICRS's features, we can conclude that the spherical power is mainly controlled by the sDI whereas it is almost independent from hDRI and $\phi_{I C R S}$. In particular, rings located farther and deeper affect more to spherical aberration than other combinations (see in figure 6.a). Regarding the cylindrical power, sDI is also controlling the overall astigmatic change (see in figure 6.b). In particular, sDI presents a nonlinear variation with hDRI, with a great variation for rings placed at 50\% depth and a null variation for rings placed at $80 \%$ depth. Despite different diameters of ICRS affect the cylindrical power, the difference between using 200 or 300 microns is negligible. Once more, the inversion of behaviour between $65 \%$ and $80 \%$ depth is observed in the interaction be- 


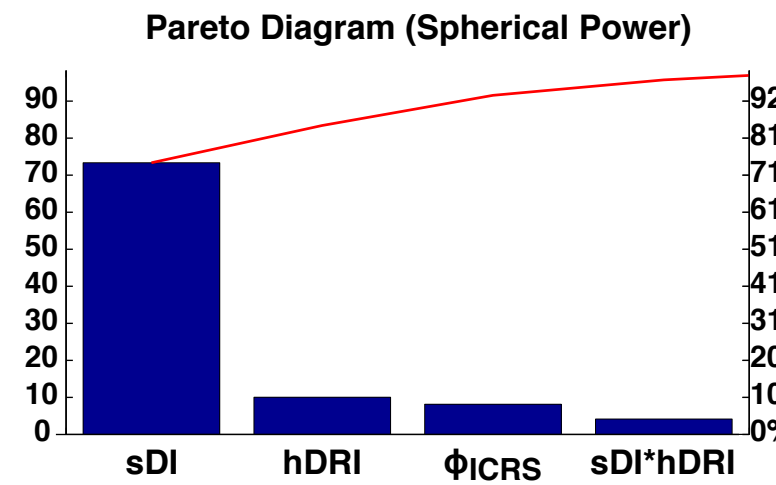

(a)
Pareto Diagram (Cylindrical Power)

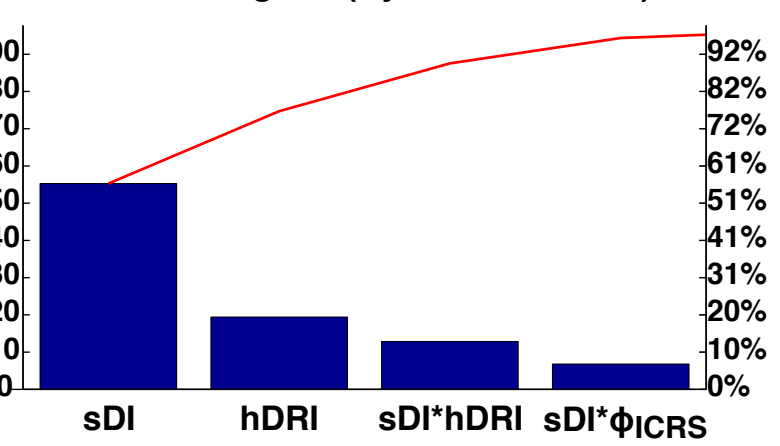

(b)
Main Effects (Spherical Power)

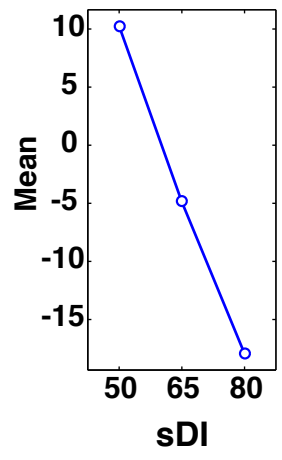

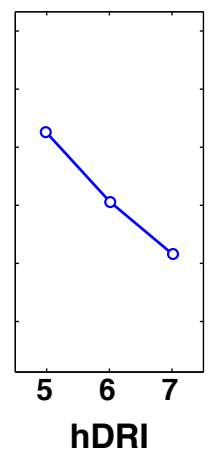

(c)

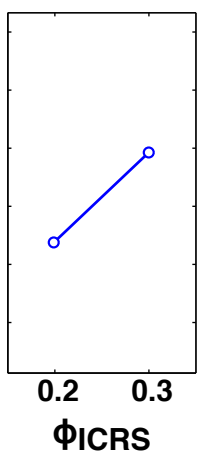

\$ICRS
Main Effects (Cylindrical Power)

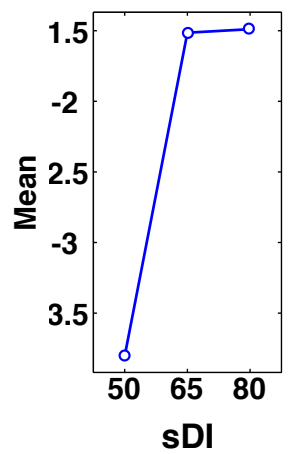

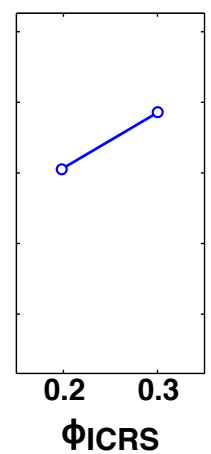

(d)

Fig. 5 Parametric analysis (1): Pareto chart and main effect of the variables under analysis in the spherical and cylindrical powers. (a-b) Influence of the variation of the variables under analysis (sDI, hDRI and $\left.\phi_{I C R S}\right)$ in the spherical (a) and the cylindrical (b) powers. (c-d) Main effect of the variation of the variables under analysis in the spherical (c) and the cylindrical (d) powers

tween sDI and hDRI, and sDI and $\phi_{I C R S}$. This fact also suggests that a mechanical change in the cornea is occurring when overpassing $65 \%$ depth, which is modifying its overall behaviour.

\subsection{Effect of ICRS in KC corneas: kinematics, mechanics, and optics}

The average $\mathrm{KC}$ cornea (see case 0 in figure 7 ) presented an apical displacement after pressurization of $0.17 \mathrm{~mm}, 3$ times bigger than in the healthy average cornea, i.e., $0.05 \mathrm{~mm}$. The apex moved back when inserting two ICRS of different diameter, 200 and 500 microns, at $80 \%$ depth. The backward movement of the smaller ICRS is larger than the one of the bigger ICRS, i.e., $\delta_{1}=-0.12$ and $\delta_{2}=-0.03 \mathrm{~mm}$ respectively (see cases 1 and 2 in figure 7.e). Results previously reported (Kahn, 2016; Kling, 2013; Lago, 2015) support the behavior of the numerical model after the insertion of ICRS. It is worth to note that, when inserting the 200-micron ICRS at 50\% depth, the apex moves forward instead of backwards with $\delta_{3}=0.12 \mathrm{~mm}$ (see case 3 in figure 7.d-e).

When the ring is placed at $80 \%$ depth, i.e., the recommended insertion depth in therapeutic treatments, the corneal stroma mostly works in a (heterogeneous) biaxial stress state that does not vastly differ from the physiological stress state of the cornea solely subjected to the eyeball's intraocular pressure (Ariza-Gracia, 2016) (see in figure 8.a). However, when the ring is placed at $50 \%$ depth, the cornea is working in a mixed stress state where the anterior stroma is compressed and the posterior stroma is in tension (see in figure 8.b). The stresses in the cone also outline the inversion of the stress field depending on the sDI. The anterior stroma bears more load whereas the posterior stroma unloads when the ring is placed to the clinical depth, and viceversa when it is placed at $50 \%$ depth (see $C_{1}$ and $C_{3}$ in figures 8.c-d). This inversion in the mechanical behaviour of 


\section{Interaction Effects (Spherical Power)}

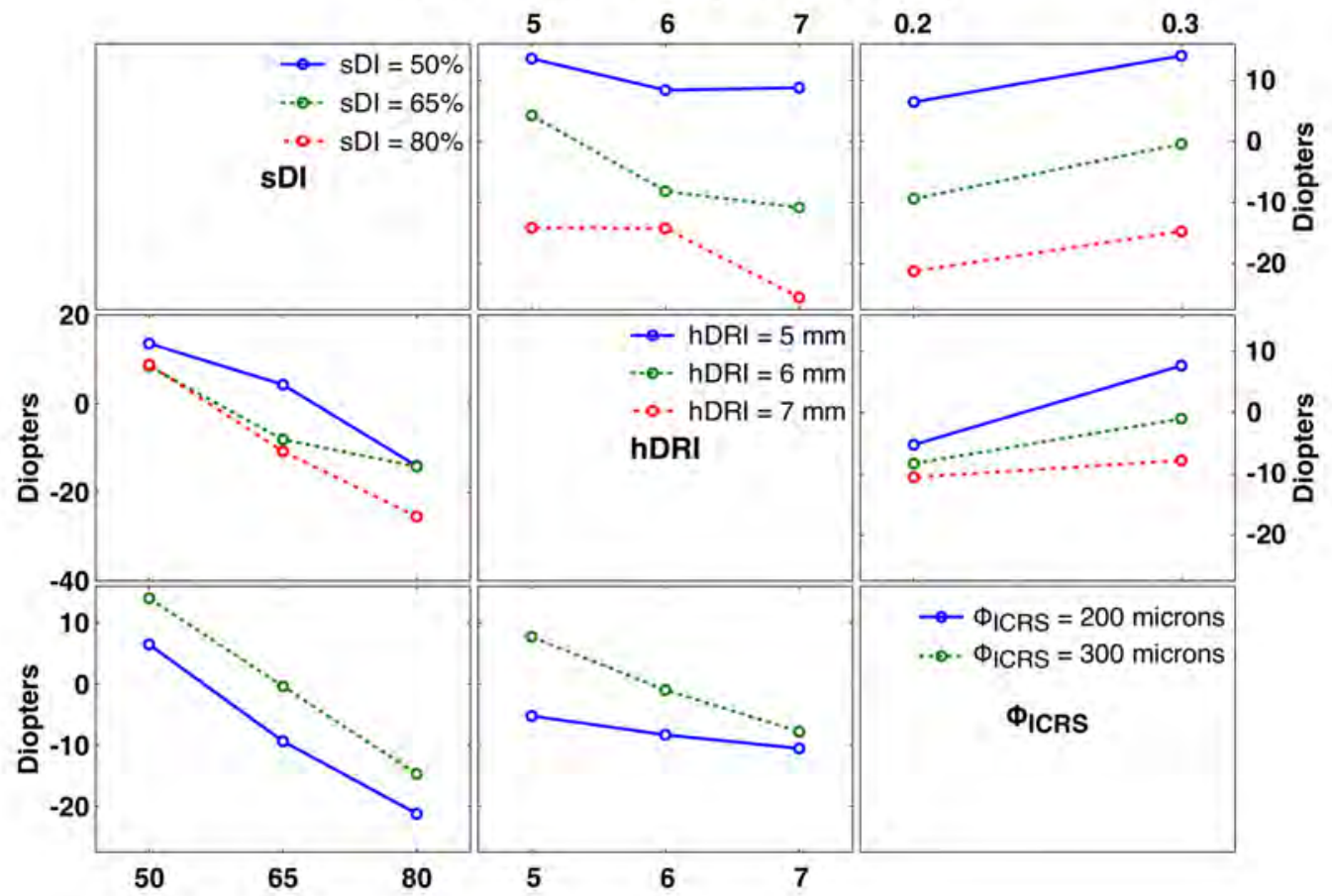

(a)

\section{Interaction Effects (Cylindrical Power)}

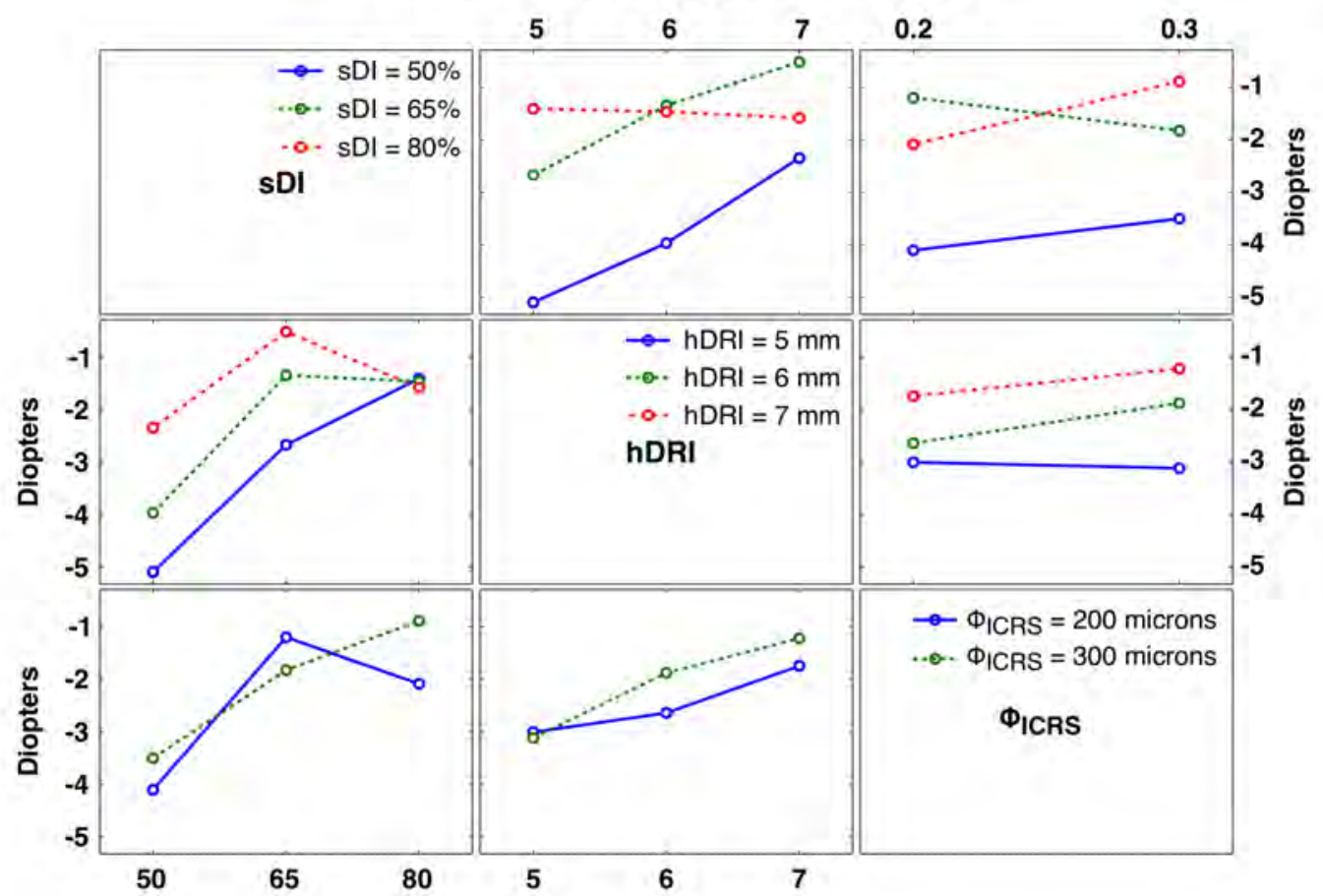

(b)

Fig. 6 Parametric analysis (2): interaction of the variables under analysis in the spherical (a) and cylindrical (b) powers. Row values are hold constant while column values present 3 levels of variation. Note: diameter of 500 microns is an outlier and was removed from the plots for the sake of clarity 


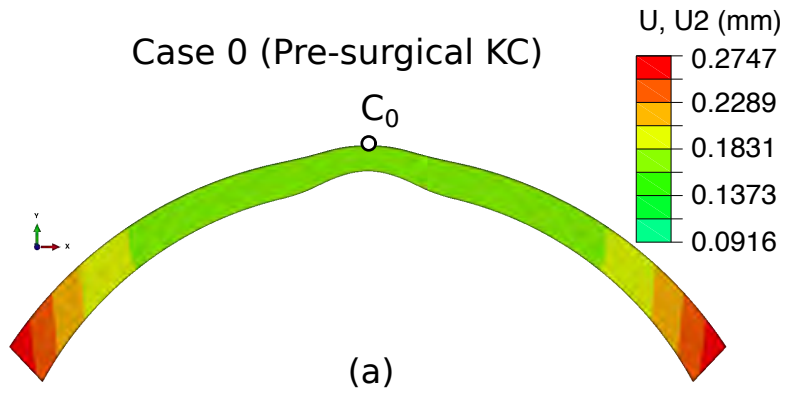

(a)
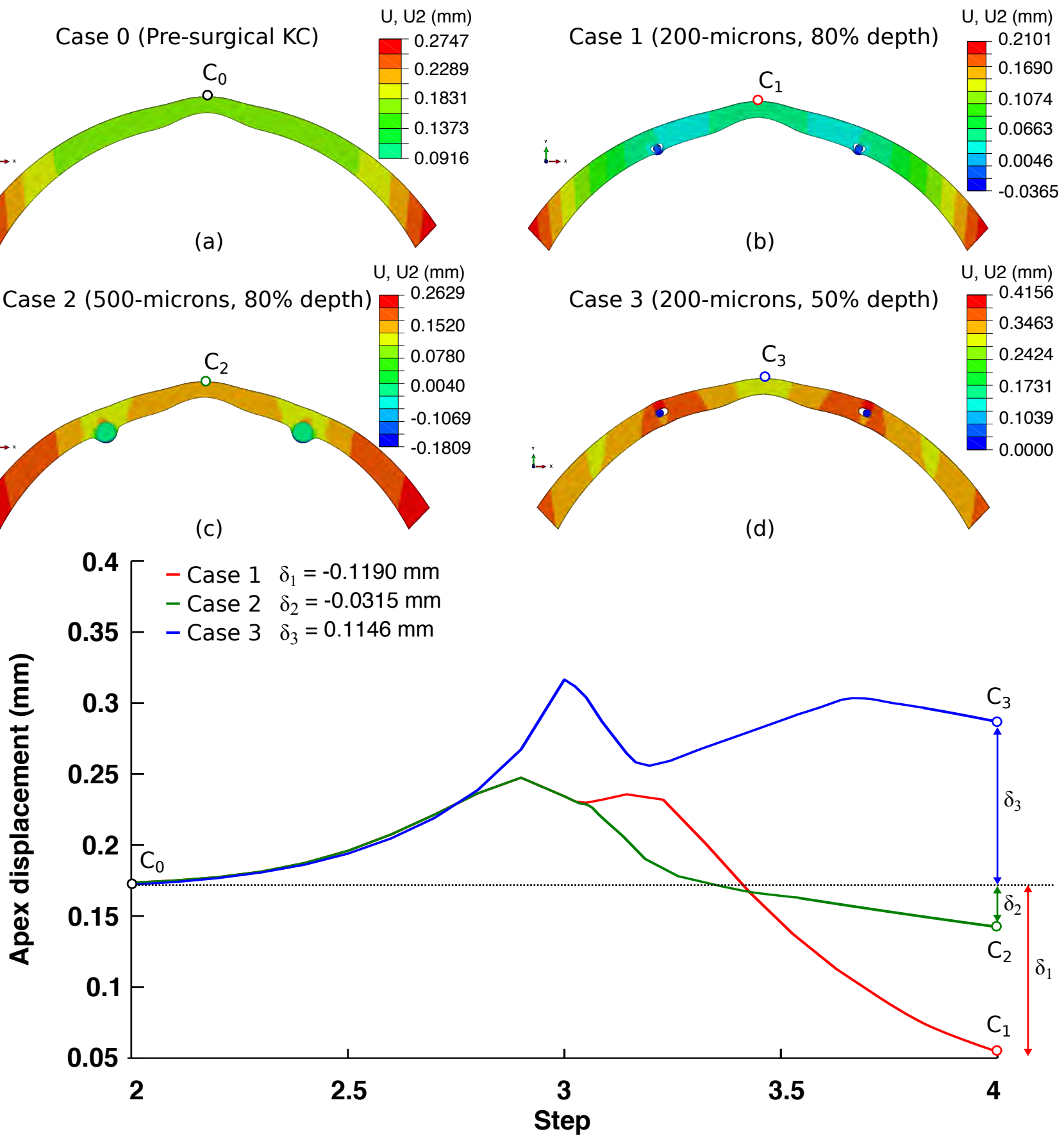

(e)

Fig. 7 Kinematics of the KC cornea: (a-d) Transverse section of the vertical displacement field of the cornea in four different cases. Case 0 is the pre-surgical configuration, Case 1 is the equilibrium configuration after the insertion of 200-micron rings at $80 \%$ depth, Case 2 is the equilibrium configuration after the insertion of $500-$ micron rings at $80 \%$ depth, and Case 3 is the equilibrium configuration after the insertion of 200-micron rings at $50 \%$ depth; (e) Displacement of the apex during the simulation procedure. Due to the pre-stress of the cornea the apex raised to $0.17 \mathrm{~mm}$. After the insertion of the rings, the apex moved backward (Case 1 and 2) or forward (Case 3) a $\delta_{i}$, decreasing or increasing the axial length of the eye. The displacement of the corneal apex for case 1 is also reported in the video provided as supplementary material (lower left panel) 
the cornea leads to a change in the corneal surface and kinematics that could explain the differences observed in the transition from $65 \%$ to $80 \%$ depth (see figures $5-8)$.

However, this hypothesis cannot be supported entirely by experimental facts (Zare, 2007; Liu, 2015; JosephColin, 2012) as rings placed at 50\% are usually rejected by the cornea. When an ICRS is inserted at $50 \%$ depth (see in figure 8.a), the tissue surrounding it is abnormally tense resulting in a higher stretch of the corneal stroma. The positive tension in the posterior stroma and close to the endothelium is causing the tissue to push the ICRS up to the epithelium, whereas the positive tension in the anterior stroma is causing the tissue to excessively stretch in the surroundings of the surgery, i.e., the hole through where ICRS are inserted. On the contrary, tensions in the anterior stroma do not appear when the ICRS is placed at $80 \%$ depth (see in figure 8.b). In this case, the ICRS is equilibrated and fastened by tensions in the posterior stroma and intraocular pressure, whereas the fibrous weak tissue of the scar in the epithelium does not support an excessive load bearing. We hypothesise that this mechanical behaviour close to the scar is promoting, among other factors, the extrusion of the ICRS.

The insertion of ICRS will also cause the corneal optics to worsen (see in tables 1, 2, and figure 9). Any ICRS generates different optical aberrations: primary, secondary, and tertiary spherical aberrations $\left(Z_{2}^{0}, Z_{4}^{0}\right.$, $\left.Z_{6}^{0}\right)$ and first and second order astigmatism $\left(Z_{2}^{2}, Z_{4}^{2}\right)$, which are low order aberrations, and high order aberrations $\left(Z_{4}^{4}, Z_{6}^{4}, Z_{6}^{6}\right)$. Optical powers and low order aberration values are shown in table 1 and high order aberration values in table 2 . In particular, the 500 micron ICRS generates the larger aberrations and affects all Zernike coefficients (see case 2 in figure 9). The $200 \mathrm{mi}-$ cron ICRS at $50 \%$ depth is generating larger high order aberrations, and affecting less to the spherical power (related to $Z_{2}^{0}$ ). On the contrary, placing it at $80 \%$ depth is barely affecting the spherical power while is inverting the sign and magnitude of the Zernike coefficient related to the cylindrical power $\left(Z_{2}^{2}\right)$. This worsening of the visual acuity of the numerical models is also supported by different clinical studies (Waleed, 2016; Fernandez-Vega, 2016; Jadidi, 2014; Torquetti, 2016) that showed an increment in astigmatism, related to the cylindrical power, although there is a slight improvement in defocus, related to the spherical power (see in tables 1 and 2).
Table 1 Optical Powers (Cylinder and Sphere) and Low Order Aberrations (LOA). Pre-surgical healthy (Pre-H) and $\mathrm{KC}$ (Pre-KC) configurations, and post-surgical KC configurations: case 1 (200-microns, 80\% Depth), case 2 (500-microns, $80 \%$ Depth), and case 3 (200-microns, 50\% Depth)

\begin{tabular}{c|ccc|cc} 
& \multicolumn{3}{|c|}{ Optical Powers } & \multicolumn{2}{c}{ LOA } \\
\hline & Sph (D) & Cyl (D) & $\phi\left(^{\circ}\right)$ & $Z_{2}^{0}\left(Z_{4}\right)$ & $Z_{2}^{2}\left(Z_{5}\right)$ \\
\hline Pre-H & -16.9 & 0.0 & 20.680 & 9.310 & 0.000 \\
Pre-KC & -21.1 & 0.0 & 0.13 & 12.060 & 0.000 \\
\hline Case 1 & -19.5 & 0.9 & 0.0 & 11.940 & -0.392 \\
Case 2 & -18.5 & -8.2 & 0.0 & 14.044 & 3.608 \\
Case 3 & -5.7 & -3.0 & 0.0 & 93.476 & 1.034 \\
\hline
\end{tabular}

\section{Discussion}

A novel template-based methodology for the simulation of the long-term effects of ICRS is presented. The main advantage of the methodology is that is suitable for a semi-automatic simulation of different clinical scenarios and allowing for setting up an optimisation procedure straightforwardly. Moreover, we go beyond the current state of the art (Kahn, 2016; Kling, 2013; Lago, 2015 ) by solving key aspects such as avoiding the subtle weakening of accounting for a gap in the pre-surgical corneal configuration, and allowing for the free threedimensional solid rigid motion of the ring after the insertion. Moreover, thanks to our opto-mechanical analysis(Ariza-Gracia, 2017), our methodology is capable of providing a comprehensive optical assessment of different post-surgical scenarios that improves the translation of in silico models to clinic.

We assumed three main simplifications since our interest is in the computational methodology and we are not presenting a numerical framework for clinical application. Nevertheless, the methodology is designed to easily cope with further and more complex characteristics.

First, the inclusion of patient-specific geometries is the next natural step and can be done by morphing the FE template into the patient-specific corneal topography provided by commercial topographers. Based on our previous experience (Ariza-Gracia, 2016), we are currently working on this line.

Second, as the previous works in the literature (Kahn, 2016; Kling, 2013; Lago, 2015), we use an isotropic hyperelastic material behaviour for the ocular tissues. Despite the sclera could be assumed as such far from the optical nerve insertion (Coudrillier, 2015), the cornea and the limbus are still anisotropic. In this line, we are working on including an anisotropic hyperelastic material behaviour with two families of orthogonal collagen fibres in the cornea and one family of circumferential collagen fibres in the limbus. This will modify the kinematics and the optics of the cornea, and the stress field in the stroma and, thus, it must be further investigated. 


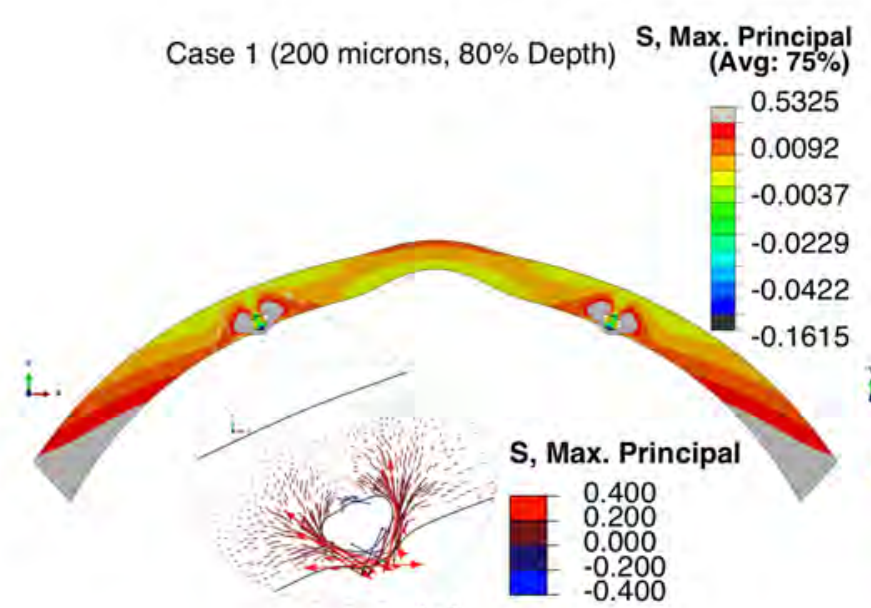

(a)

Relaxation Anterior Cornea

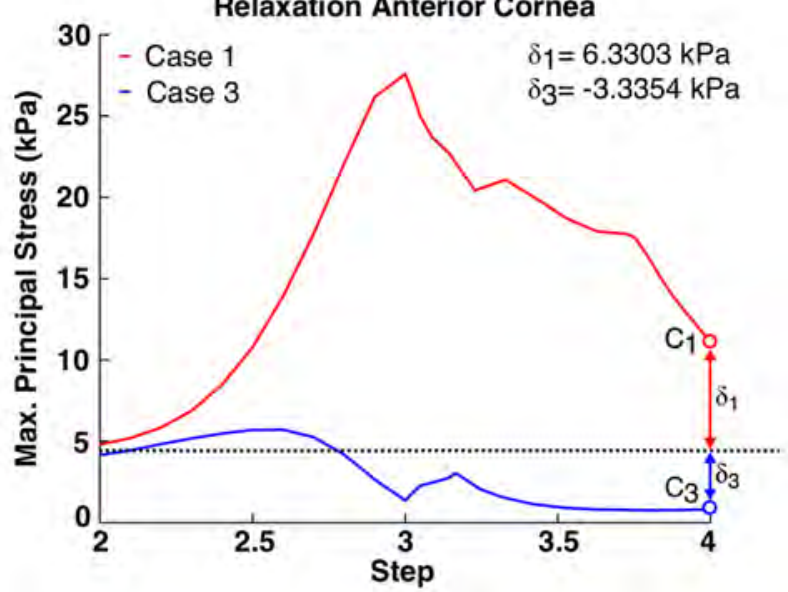

(c)

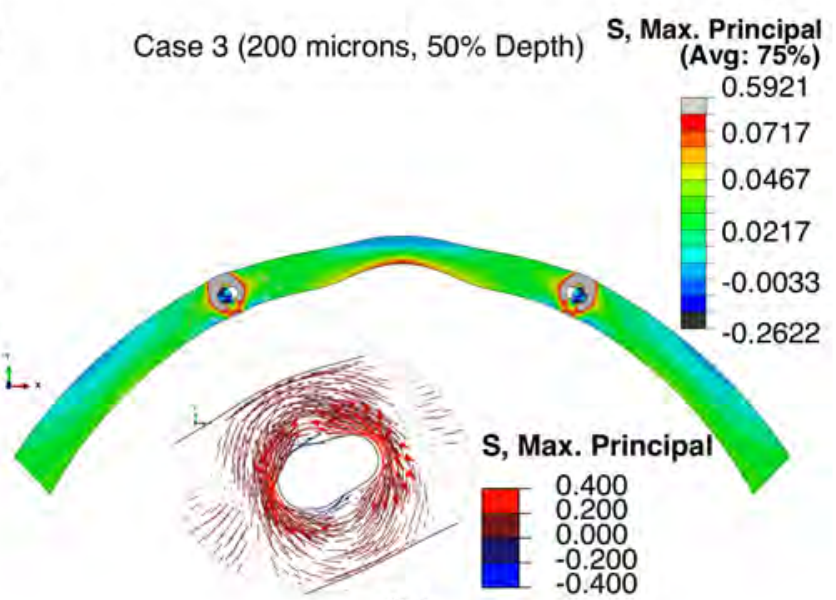

(b)

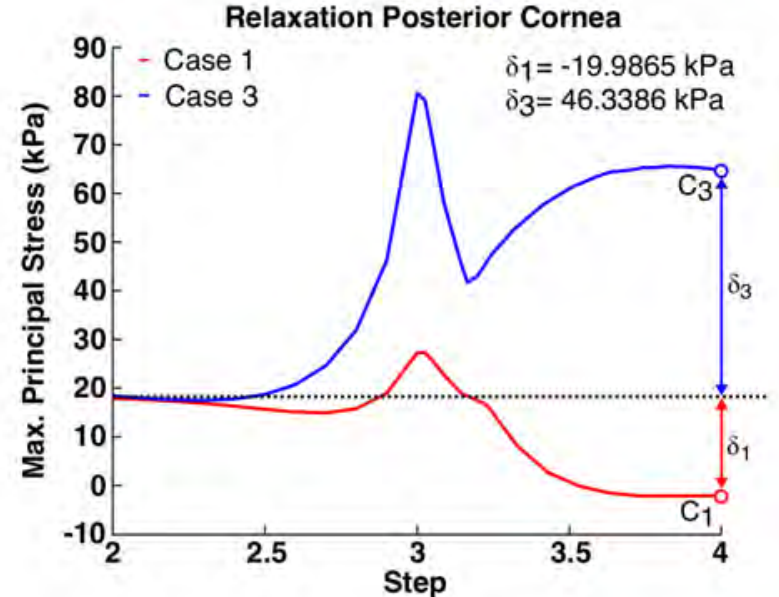

(d)

Fig. 8 Mechanics of the KC cornea. (a) Stress field (MPa) in the stroma for Case 1 (200 micron and $80 \%$ depth) in which cornea presents an heterogeneous biaxial stress state. (a-zoom) Principal stresses (MPa) in the surroundings of the incision. Above the ICRS there is almost no stress while under the ring there is maximum traction. This state of stress will fasten the ring in the incision; (b) Stress field (MPa) in the stroma for Case 3 (200 micron and 50\% depth) in which cornea presents a mixed stress state with compression in the anterior surface and tension in the posterior surface. (b-zoom) Principal stresses $(\mathrm{MPa})$ in the surroundings of the incision. There is traction above and under the ring. This state of stress could tear up the fibrous tissue of the scar in the epithelium while pushing the ring out of the incision from the posterior stroma; (c) Relaxation of the cone in the anterior cornea. The cone is more tensioned in case 1 while it is relaxed in case 3 ; (d) Relaxation of the cone in the posterior cornea. The cone is more tensioned in case 3 while it is relaxed in case 1 . The gradient of the maximum principal stress around the ICRS (upper right panel) and the temporal evolution of the maximum principal stress in the anterior and posterior cone (lower right panel) for case 1 is also reported in the video provided as supplementary material

Table 2 High Order Aberrations (HOA). Pre-surgical healthy (Pre-H) and KC (Pre-KC) configurations, and post-surgical KC configurations: case 1 (200-microns, 80\% Depth), case 2 (500-microns, 80\% Depth), and case 3 (200-microns, 50\% Depth)

\begin{tabular}{c|cccccc} 
& $Z_{4}^{0}\left(Z_{12}\right)$ & $Z_{4}^{2}\left(Z_{13}\right)$ & $Z_{4}^{4}\left(Z_{14}\right)$ & $Z_{6}^{0}\left(Z_{24}\right)$ & $Z_{6}^{4}\left(Z_{26}\right)$ & $Z_{6}^{6}\left(Z_{27}\right)$ \\
\hline Pre-H & 0.605 & 0.0 & 0.0 & 0.023 & 0.0 & 0.0 \\
Pre-KC & -1.950 & 0.0 & 0.0 & 2.897 & 0.0 & 0.0 \\
\hline Case 1 & -3.397 & 0.299 & -0.175 & 3.404 & 0.076 & -0.082 \\
Case 2 & -4.584 & -0.773 & 2.457 & 3.109 & -0.708 & 1.584 \\
Case 3 & -3.149 & 0.191 & 0.788 & 3.699 & 0.105 & 0.604 \\
\hline
\end{tabular}




\section{Post Surgical Zernike Coefficients (Keratoconus Eye)}

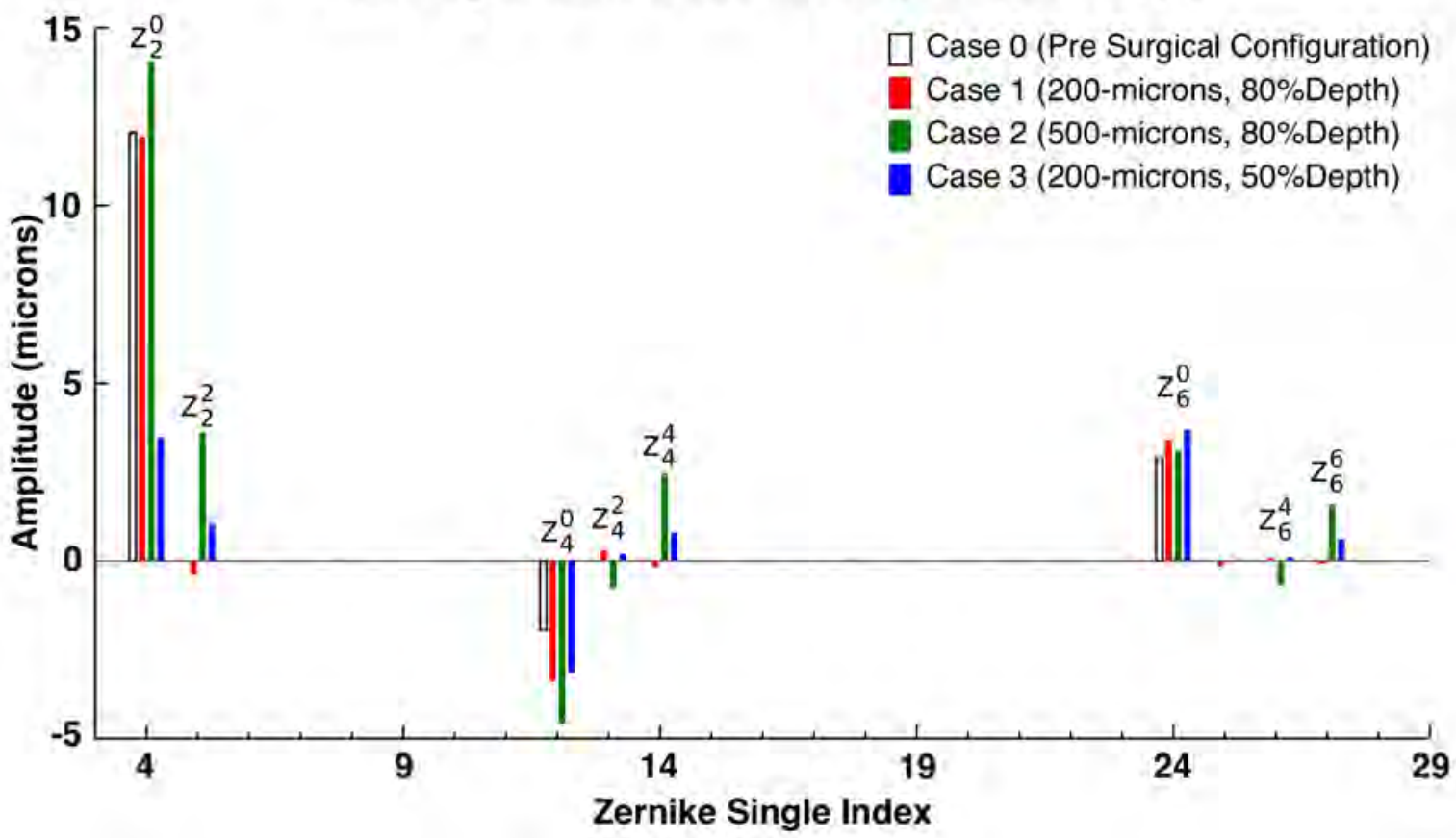

Fig. 9 Zernike coefficients of the KC corneas. Pre-surgical configuration is depicted in black and white. The inclusion of the rings worsened the spherical aberrations $\left(Z_{2}^{0}, Z_{4}^{0}\right.$, and $\left.Z_{6}^{0}\right)$ while introducing astigmatism $\left(Z_{2}^{2}\right.$, and $\left.Z_{4}^{2}\right)$ and high order aberrations $\left(Z_{4}^{4}, Z_{6}^{4}\right.$, and $\left.Z_{6}^{6}\right)$

Third, we use circular-shaped ICRS with a diameter that is equivalent to the maximum dimensions of commercial triangular or hexagonal rings (see in figure 2). In terms of methodology, we ensure that the incision is big enough to house any typology of ICRS. The next steps of our research will focus on including triangular and hexagonal rings and on solving the contact with the incisions.

A final remark regarding the limitations encompasses the semi-automatisation of the methodology. As it is a template-based approach, we are constrained by the number of $\mathrm{FE}$ templates available, which are discrete and do not allow for continuous inspection of the different parameters. In the future, we will fully automatise the procedure allowing to set a complete clinical scenario in a non-supervised process.

The methodology generates outcomes that behave as expected based on clinical findings (Waleed, 2016; Fernandez-Vega, 2016; Jadidi, 2014; Torquetti, 2016). The $3^{k}$ full-factorial analysis outlined this physiological behaviour through different key findings (see in figures 5 and 6). First, the sDI is the most influential parameter in the post-surgical visual acuity of the patient (Kahn, 2016; Barbara, 2015), representing between a 50 and a $70 \%$ of the impact in the spherical (see in figure 5.a) and cylindrical powers (see in figure 5.b). Second, the farther the hDRI, the higher the impact on the spherical power but the lower in the cylindrical power (Abdelmassih, 2017). Third, the $\phi_{I C R S}$ does not present a large variability when using 200 or 300 microns. Moreover, 500 micron rings should not be used in diameters lower than $7 \mathrm{~mm}$ as they are causing incredible aberrations.

The kinematic and optical behaviours are also in line with those reported in the literature (Kahn, 2016; Kling, 2013) and support the physiological behaviour of the model and the methodology. In terms of kinematics, when the ICRS is inserted at $80 \%$ depth, i.e., the insertion depth used in clinic, there is a move backward of the corneal apex that changes the axial length of the eye and the curvature (see in figure 7). Optically, the insertion of the ICRS seems to regularise the corneal surface as the spherical power decreases. However, it worsens the overall visual acuity of the patient and, in particular, astigmatism and high order aberrations.

Interestingly, there is a transition zone between 65 and $80 \%$ depth where the mechanical behaviour of the cornea completely changes. When the rings are inserted close to the anterior stroma, the cornea tends to slightly bend as the anterior surface is in compression and the posterior surface is in tension. However, when the rings are inserted close to the endothelium, the corneal stroma 
is still working in a (heterogeneous) biaxial stress state similar to the physiological stress state of the cornea (Ariza-Gracia, 2016).

As the evolution of $\mathrm{KC}$ is suggested to be stressdriven (Zare, 2007; JosephColin, 2001; Rabinowitz, 1998), we hypothesise that its progression could be differently affected depending on where it spatially develops. If the $\mathrm{KC}$ rises from the posterior surface and propagates towards the epithelium, it would be advisable to place the ICRS at $80 \%$ depth as it would relax the posterior cornea and stretch the anterior cornea. However, if the origin of the pathology is in close to the epithelium, it would be advisable to place a ring at $50 \%$. However, rings placed at $50 \%$ depth presented corneal extrusion of the ICRS in clinic(Zare, 2007; Liu, 2015; JosephColin, 2012).

Thanks to our study of the mechanical influence of the stromal depth of insertion (sDI), we also hypothesise that corneal extrusion is related to the gradient of principal stresses in the surroundings of the incision (see in figure 8). When the ICRS is placed at $50 \%$ depth, the positive stresses above the ring tend to tear up the tissue in the surroundings of the scar through where the ring was inserted. At the same time, the positive stresses under the ICRS tend to stretch the tissue and push the ring up. In combination, both behaviours could cause the fibrous tissue of the scar to tear up and the corneal stroma to expel the ring. Although we are using an isotropic material and further research must be done, this is the first mechanics-based hypothesis that, to the best of our knowledge, explains the corneal extrusion and is supported by an in silico study.

In conclusion, the methodology proposed is suitable for simulating the long-term mechanical and optical effects of ICRS insertion. Currently, we are investing further efforts in extending the methodology to cope with patient-specific geometries, to instruct material models with anisotropy, to use different commercial rings, and to achieve a fully automatic methodology with continuous inspection of the ICRS's parameters involved in the surgery.

Acknowledgements Funding: This work was supported by the Spanish Ministry of Economy and Competitiveness (Projects DPI2014-54981-R and DPI2017-84047-R), Department of Industry and Innovation (Government of Aragón) and European Social Fund 2014-2020 (FSE-DGA T88). J.Flecha was supported by the Spanish Ministry of Economy and Competitiveness (BES-2015-073630). M.Á. Ariza-Gracia was supported by the Swiss Government through the ESKAS program (ESKAS-No: 2016.0194. Federal Commission for Scholarships for Foreign Students FCS, Switzerland)

Conflict of interest: The authors declare that they have no conflict of interest.

\section{References}

Abdelmassih Y., El Khoury S., Chelala E., Slim E., Cherfan C.G., Jarade E (2017) Toric ICL implantation after sequential intracorneal ring segments implantation and corneal cross-linking in keratoconus: 2-year follow-up. Journal of Refractive Surgery 33(9):610-616. doi:https://doi.org/10. 3928/1081597X-20170621-02.

Alió J.L., Artola A., Jassanein A., Haroun A., Galal A (2005) One or 2 intacs segments for the correction of keratoconus. Journal of Cataract and Refractive Surgery 31:943-953.

Alió J.L., Shabayek M.H., Artola A (2006) Intracorneal ring segments for keratoconus correction: Long-term follow-up. Journal of Cataract and Refractive Surgery 32:978-985.

Akaishi L., Tzelikis P.F., Raber I.M (2004) Ferrara intracorneal ring implantation and cataract surgery for the correction of pellucid marginal corneal degeneration. Journal of Cataract and Refractive Surgery 30(11):2427-2430. doi:http://dx.doi. org/10.1016/j.jcrs.2004.04.047.

Ariza-Gracia M. Á., Zurita J., Piñero D.P., RodríguezMatas J.F., Calvo B (2015) Coupled biomechanical response of the cornea assessed by non-contact tonometry. A simulation study. PlosOne 10(3):1-10. Ariza-Gracia M. Á. , Zurita J., Piñero D.P., Calvo B., Rodríguez-Matas J.F (2016) Automatized patientspecific methodology for numerical determination of biomechanical corneal response. Annals of Biomedical Engineering 44(5):1753-1772. doi:10.1007/ s10439-015-1426-0.

Ariza-Gracia M. Á., Ortillés A., Cristóbal J., Rodríguez-Matas J.F., Calvo B (2017) A numericalexperimental protocol to characterize corneal tissue with an application to predict astigmatic keratotomy surgery. Journal of the Mechanical Behavior of Biomedical Materials 74(2017):304-314.

Auffarth G.U., Wang L., Völcker H.E (2000) Keratoconus evaluation using the orbscan topography system. Journal of Cataract and Refractive Surgery 26(2)222-228. doi:https://doi.org/10. 1016/S0886-3350 (99)00355-7.

Barbara R., Barbara A., Naftali M (2015) Depth evaluation of inteded vs intacts intrastromal ring segments using opitcal coherence tomography. Eye 30:102-110.

Benoit A., Latour G., Schanne-Klein M.C., Allain J.M (2015) Simultaneous microstructrual and mechanical characterzation of human corneas at increasing pressure. Journal of the Mechanical Behavior of Biomedical Materials 60(2016):93-105. 
Colin J., Cochenre B., Savary G., Malet F., HolmesHiggin D (2001) INTACS inserts for treating keratoconus. American Academy of Ophtalmology 108(8):1409-1414.

Colin J., Kiliç A. (2012) Surgical techniques for ICRS Implantation. Cataract and Refractive Surgery Today Europa:23-24.

Coskunseven E., Kymionis G.D., Tsiklis N.S., Atun S., Arslan E., Jankov M.R., Pallikaris I.G. (2008) Oneyear results of intrastromal corneal ring segment implantation (keraring) using femtosecond laser in patients with keratoconus. American Journal of Ophthalmology 145(5):775-779.e1. doi:https://doi. org/10.1016/j.ajo.2007.12.022.

Coudrillier B., Pijanka J., Jefferys J., Sorensen T., Quigley H.A., Boote C., Nguyen T.D (2015) Effects of age and diabetes on scleral stiffness. Journal of Biomechanical Engineering 137(7):1-10. doi: $10.1115 / 1.4029986$.

Dan Z R., Timothy J A., Marine G., Raksha U., Ronald H S (2017) Keratoconus: Recent Advances in Diagnosis and Treatments Ch.12:141-150.

Fangjun B., Brendan G., QinMei W., Ahmed E (2016) Consideration of corneal biomechanics in the diagnosis and management of keratoconus: is it important? Eye and Vision 2016:3-18. doi:http://dx. doi.org/10.1186/s40662-016-0048-4.

Fernandez-Vega L., Lisa C., Poo-López A., MadridCosta D., Merayo-Lloves J., Alfonso J.F (2016) Intrastromal corneal ring segment implantation in 409 paracentral keratoconic eyes. Cornea 35(11):14211426.

Garcia-Porta N., Fernandes P., Queiros A., SalgadoBorges J., Parafita-Mato M., González-Méijome J.M (2014) Corneal biomechanical properties in different ocular conditions and new measurement techniques. ISRN Ophthalmology 2014:1-20.

Garzón N., Galán F.P (2013) Orbscan: Topographical maps. Gaceta Optica 420:24-28.

Gomes J.A., Tan D., Rapuano C.J., Belin M.W., Renato Ambrósio J., Guell J.L., Malecaze F., Nishida K., Sangwan V.S (2015) Global consensus on keratoconus and ectasic disease. Cornea 34(4):359-369.

Guang-Ming Dai G (2008) Wavefront optics for vision correction. SPI press, Bellingham, Washington USA, Washington.

Guarnieri F.A., Ferrara P., Torquetti L (2015) Biomechanics of Additive Surgery: Intracorneal Rings Ch.5:73-107. doi:http://dx.doi.org/10.1007/ 978-1-4939-1767-9.

Hernández-Gómez G., Malacara-Doblado D., Malacara-Hernández Z., Malacara-Hernández D (2014) Modal processing of hartmann and shack-hartmann patterns by means of a least squares fitting of the transverse aberrations. Applied Optics 53(1):7422-7434. doi:http://dx.doi.org/10.1364/A0.53.007422.

Hong J., Xu J., Wei A., Deng S.X., Cui X., Yu X., Sun X. (2013) A new tonometer? the corvis st tonometer: clinical comparison with noncontact and goldmann applanation tonometers. Investigative Ophthalmology and Visual Science 54:659-665.

Jadidi K., Mosavi S.A., Nejat F. , Naderi M., Janani L., Serahati S. (2014) Intrastromal corneal ring segement implantation (keraring $355^{\circ}$ ) in patients with central keratoconus: 6-month follow-up, Hindawi Publishing Corporation. Journal of Ophthalmology 2015:18. doi:http://dx.doi.org/10.1155/2015/916385.

Kahn S.N., Shiakolas P.S (2016) To study the effects of intrastromal corneal ring geometry and surgical condictions on the postsurgical outcomes through finite element analysis. Journal of Mechanics in Medicine and Biology 16(7):1-16. doi:http://dx.doi.org/ 10.1142/S0219519416501013.

Kling S., Marcos S (2013) Finite-element modeling of intrastromal ring segment implantation into a hyperelastic cornea. Investigate Ophthalmology and Visual Science 54(1):881-889.

Lago M., Rupérez M.J., Monserrat C., MartínezMartínez F., Martínez-Sanchís S., Larra E., DíezAjenjo M., Peris-Martínez C. (2015) Patient-specific simulation of the intrastromal ring segment implantation in corneas with keratoconus. Journal of the Mechanical Behavior Biomedical Materials 51:260-268. doi:https://doi.org/10.1016/j. jmbbm.2015.07.023.

Lakshminarayanan V., Fleck A. (2011) Zernike polynomials: a guide. Journal of Modern Optics 58(7):545561. doi:10.1080/09500340.2011.554896.

Liu X.L., Li P.H., Fournie P., Malecaze F (2015) Investigation of the efficency of intrastromal ring segments with cross-linking using different sequence and timing for keratoconus. International Journal of Ophthalmology 8(4):703-708. doi:http://dx.doi.org/ 10.3980/j.issn.2222-3959.2015.04.11.

Malacara D., Malacara Z (2003) Handbook of Optical Design. 2nd Edition, Marcel Dekker.

Montgomery D.C (2001) Desgin and Analysis of Experiments, 5th Edition, John Wiley and Sons, 1997.

Navarro R., González L., Hernández J.L (2006) Optics of the average normal cornea from general and canonical representations of its surface topography, Journal of the Optical Society of America 23(2):219-32. doi:10.1364/JOSAA.23.000219.

O'Brat D (2017) Keratoconus: Recent Advances in Diagnosis and Treatments Ch. 19:219-238. 
Pandolfi A., Vasta M. (2012) Fiber distributed hyperelastic modeling of biological tissues. Mechanics of Materials 44(Supplement C):151-162, microstructures and anisotropies. doi:https://doi.org/10. 1016/j.mechmat.2011.06.004.

Peris-Martínez C., Cisneros Lanuza Á.L (2014) Biomechanics and Architecture Corneal Ch.3:25-34.

Piñero D.P., Alió J.L., Kady B.E., Coskunseven E., Morbelli H., Uceda-Montanes A., Maldonado M.J., Cuevas D., Pascual I (2009) Refractive and aberrometric outcomes of intracorneal segments for keratoconus: Mechanical versus femtosecond-assited procedures. American Academy of Ophtalmology 116:1675-1687.

Rabinowitz Y.S (1998) Keratoconus. Survey of Ophthalmology 42(4):297-319. doi:http://dx.doi. org/10.1016/S0039-6257(97)00119-7.

Ramez B., Andrew MJ T., Parwez H., David F A., Adel B (2017) Keratoconus: Recent Advances in Diagnosis and Treatments Ch.3:13-23.

Scarcelli G., Yun S. H (2017) Keratoconus: Recent Advances in Diagnosis and Treatments. Chap. 14:167173.

Shabayek M.H., Alió J.L (2007) Intrastromal corneal ring segment implantation by femtosecond laser for keratoconus correction. American Academy of Ophtalmology 114:1643-1652.

Sherwin T., Ismail S., Loh I.P., McGhee J.J (2017) Keratoconus: Recent Advances in Diagnosis and Treatments Ch.4:25-41.

Suiter B.G., Twa M.D., Ruckhofer J., Schanzlin D.J (2000) A comparison of visual acuity, predictability and visual function outcomes after intracorneal ring segments and laser in situ keratomileusis. Transactions of the America Ophthalmological Society 98: $51-57$.

Thibos L.N., Hong X., Bradley A., Applegate R.A (2004) Accuracy and precision of objective refraction from wavefront aberrations. Journal of Vision 4(4):329-351. doi:10.1167/4.4.9.

Torquetti L., Arce C., Merayo-Lloves J., Ferrara G., Ferrara P., Signorelli B., Signorelli A. (2016) Evaluation of anterior and posterior surfaces of cornea using a dual scheimpflug analyzer in keratoconus patients implanted with intrastromal corenal ring segements. International Journal of Ophthalmology 9(9):1283-1288. doi:http://dx.doi.org/10. 18240/ijo.2016.09.08.

Torquetti L., Ferrara G., Almeida F., Cunha L., Ferrara P., Merayo-Lloves J. (2013) Clinical outcomes after intrastromal corneal ring segments reoperation in keratoconus patients. International Journal of Ophthalmology 6(6):796-800. doi:http://dx.doi.org/
$10.3980 / j$. issn. 2222-3959.2013.06.10.

Vega-Estrada A., Alió J.L (2016) The use of intracorneal ring segments in keratoconus. Eye and Vision 2016:3-8.

Al-Tuwairqui W.S., Osuagwu U.L., Razzouk H., AlHarbi A., Ogbuehi K.C (2016) Clinical evaluation of two types of intracorneal ring segments (ICRS) for keratoconus. International Ophthalmology 37(5):1185-1198. doi:http://dx.doi.org/10. 1007/s10792-016-0385-2.

Zare M.A., Hashemi H., Salari M.R (2007) Intracorneal ring segment implantation for the management of keratoconus: safety and efficacy. Journal of Cataract and Refractive Surgery 33(11):18861891. doi:http://dx.doi.org/10.1016/j.jcrs. 2007.06 .055$. 